\title{
Control and Stabilization of an Underactuated Surface Vessel
}

\author{
Mahmut Reyhanoglu \\ Department of Applied Mathematics \\ University of Twente \\ Enschede, The Netherlands \\ m.reyhanoglu@math.utwente.nl
}

\begin{abstract}
This paper studies the problem of controlling the planar position and orientation of an autonomous surface vessel using two independent thrusters. It is first shown that although the system is not asymptotically stabilizable to a given equilibrium configuration using a time-invariant continuous feedback, it is strongly accessible and small-time locally controllable at any equilibrium. Time-invariant discontinuous feedback control laws are then constructed to asymptotically stabilize the system to the desired configuration with exponential convergence rates. A simulation example is included to demonstrate the results.
\end{abstract}

\section{Introduction}

In the past few years, there has been a considerable amount of interest in the control of underactuated mechanical systems, i.e. systems with fewer inputs than degrees of freedom. The possibility of controlling a system with fewer than the typical number of actuators is indeed appealing, for it allows to reduce cost, weight as well as the occurrence of component failures. However, in general, underactuated systems present challenges which are not found in systems with full control. Controllability, for instance, which is usually implied in systems with full control, is not easy to determine in an underactuated system. Control synthesis for an underactuated system is also more complex than it is for a system with full control. While many interesting techniques and results have been presented for underactuated systems ([3], [6], [8], [11], [13], [14], [16]), the control of these systems still remains an open problem.

This paper considers the problem of controlling the planar position and orientation of an autonomous surface vessel using two independent thrusters. The dynamics of this underactuated system is complex enough to yield a rich source of control problems, yet simple enough to permit a complete mathematical analysis. It has been shown in [19] that underactuated vehicles do not satisfy Brockett's necessary condition [5] if the unactuated dynamics contain no gravitational field component and, hence, in this case, these vehicles are not asymptotically stabilizable to a given equilibrium configuration via time-invariant continuous feedback laws.
In this paper, it is first shown that although the system is not asymptotically stabilizable to a desired equilibrium configuration using a time-invariant continuous feedback, it is strongly accessible and small-time locally controllable at any equilibrium and, hence, the system is asymptotically stabilizable to a desired equilibrium using time-invariant discontinuous feedback laws. Discontinuous feedback laws are then constructed to achieve asymptotic stabilization. The methodology followed in the construction of the discontinuous feedback laws is based on first transforming the system into a discontinuous one in which the design of feedback laws is easily carried out. Then, transforming back into the original coordinates yields discontinuous feedback laws which asymptotically stabilize the original system with exponential convergence rates. This construction procedure is related to the approaches proposed in [2], [12] for the stabilization of nonholonomic systems.

The organization of this paper is as follows. In Section 2, the mathematical model describing the dynamics of a surface vessel with two independent thrusters is introduced. Controllability and stabilizability results are presented in Section 3. Discontinuous feedback laws are derived in Section 4. In Section 5, a simulation example is included. Finally, Section 6 consists of a summary of the main results.

\section{Mathematical Model}

Consider the problem of controlling the Cartesian position and orientation of a surface vessel with two independent propellers as shown in Figure 1. The kinematic model which describes the geometrical relationship between the earth-fixed (I-frame) and the vehiclefixed (B-frame) motion is given as

$$
\begin{aligned}
& \dot{x}=v_{x} \cos \psi-v_{y} \sin \psi, \\
& \dot{y}=v_{x} \sin \psi+v_{y} \cos \psi, \\
& \dot{\psi}=\omega_{z},
\end{aligned}
$$

where $(x, y)$ denotes the I-frame position of the center of mass of the vehicle, $\psi$ denotes the orientation angle; $\left(v_{x}, v_{y}\right)$ and $\omega_{z}$ are the linear and angular velocities of the vehicle in the B-frame. For simplicity, assume that the origin of the B-frame is located at the center of mass 
of the vehicle. Also assume that the vehicle is neutrally buoyant. Then the dynamic equations of motion of the vehicle can be expressed in the $B$-frame as:

$M \dot{\nu}+C(\nu) \nu+D(\nu) \nu=\tau$

where $\nu=\left(v_{x}, v_{y}, \omega_{z}\right)^{T}$ denotes the velocity vector, $\tau=\left(F_{x}, 0, T_{z}\right)^{T}$ denotes the vector of external force and torque generated by the two propellers. $M \in R^{3 \times 3}$ is the inertia matrix, including added mass; and $C(\nu) \in$ $R^{3 \times 3}$ and $D(\nu) \in R^{3 \times 3}$ denote the Coriolis/centrifugal and the damping matrices, respectively. The reader is referred to [7] for the general formulation. Assuming that $M$ is constant and diagonal, and neglecting the hydrodynamic damping terms of order higher than one, the dynamic equations of motion can be rewritten in component form as

$m_{11} \dot{v}_{x}-m_{22} v_{y} \omega_{z}+d_{11} v_{x}=F_{x}$

$m_{22} \dot{v}_{y}+m_{11} v_{x} \omega_{z}+d_{22} v_{y}=0$

$m_{33} \dot{\omega}_{z}+\left(m_{22}-m_{11}\right) v_{x} v_{y}+d_{33} \omega_{z}=T_{z}$

where $m_{i i}, d_{i i}, i=1,2,3$, are positive constants. In earth-fixed coordinates equation (6) takes the form of a second-order nonholonomic constraint, i.e. a nonintegrable relation involving not only the generalized coordinates and velocities but also the generalized accelerations. In contrast to first-order nonholonomic case (see e.g. [4]), a second-order nonholonomic constraint does not reduce the dimension of the state space. A set of three independent configuration variables and three kinematic variables is required to completely specify the state of the system.

Define the state variables

$x_{1}=\psi$,

$x_{2}=x \cos \psi+y \sin \psi$

$x_{3}=-x \sin \psi+y \cos \psi$,

$x_{4}=v_{y}$,

$x_{5}=\omega_{z}$

$x_{6}=v_{x}$

so that the state equations are given by

$\dot{x}_{1}=x_{5}$

$\dot{x}_{2}=x_{6}+x_{3} x_{5}$

$\dot{x}_{3}=x_{4}-x_{2} x_{5}$

$\dot{x}_{4}=-\alpha x_{4}-\beta x_{5} x_{6}$

$\dot{x}_{5}=u_{1}$

$\dot{x}_{6}=u_{2}$, where $\alpha=d_{22} / m_{22}, \beta=m_{11} / m_{22}$ and

$u_{1}=\left(T_{z}-d_{33} \omega_{z}+\left(m_{11}-m_{22}\right) v_{x} v_{y}\right) / m_{33}$,

$u_{2}=\left(F_{x}+m_{22} v_{y} \omega_{z}-d_{11} v_{x}\right) / m_{11}$.

In the next section, we will consider the system (8)-(13) and study its controllability and stabilizability properties.

\section{Controllability and Stabilizability}

Equations (8)-(13) define a nonlinear control system of the form

$\dot{x}=f(x)+\sum_{i=1}^{2} g_{i}(x) u_{i}$,

where $x \in \mathbf{M}=S \times R^{5}$ is the state; and $f$ and $g_{i}, i=$ 1,2 , are the drift and control vector fields. Note that the set of equilibrium solutions corresponding to $u=0$ is given by the equilibrium manifold

$\mathbf{M}^{e}=\left\{x \in \mathbf{M} \mid x_{4}=x_{5}=x_{6}=0\right\}$.

It is easily verified that the linearization of the equations (8)-(13) about an equilibrium $x^{e}$ has an uncontrollable eigenvalue at the origin. This implies that a nonlinear analysis is necessary in order to characterize the controllability and stabilizability properties of the system. Note that since the linearization of the equations (8)-(13) is not stabilizable, the system cannot be exponentially stabilized at an equilibrium using smooth feedback [20]. Moreover, it is easy to see that the system does not satisfy Brockett's necessary condition [5] and hence it is not asymptotically stabilizable to a desired equilibrium solution using time-invariant continuous feedback.

We now consider the nonlinear control system (8)(13) and employ certain results of nonlinear control theory. We refer the reader to [10] and [17] for the relevant controllability definitions and results used in the subsequent development.

The following results characterize the controllability and stabilizability properties of the underactuated vehicle dynamics described by equations (8)-(13).

Proposition 1: The underactuated vehicle dynamics described by equations (8)-(13) is strongly accessible on M.

Proof: Consider the system (8)-(13). Since the vector fields

$g_{1}, g_{2},\left[f, g_{1}\right],\left[f, g_{2}\right],\left[g_{2},\left[f, g_{1}\right]\right],\left[\left[f, g_{2}\right],\left[f, g_{1}\right]\right]$

span a six dimensional space at any point $x \in \mathbf{M}$, the strong accessibility Lie algebra rank condition is satisfied at any point. Hence the system is strongly accessible on $\mathbf{M}$.

Proposition 2: The underactuated vehicle dynamics described by equations (8)-(13) is small time locally controllable at any equilibrium $x^{e} \in \mathbf{M}^{e}$. 
Proof: Consider the system (8)-(13). Now, following Sussmann [17], let $\operatorname{Br}(\mathrm{X})$ denote the smallest Lie algebra of vector fields containing $f, g_{1}$, and $g_{2}$ and let $\mathrm{B}$ denote any bracket in $\operatorname{Br}(\mathrm{X})$. Let $\delta^{0}(B), \delta^{1}(B)$ and $\delta^{2}(B)$ denote the number of times $f, g_{1}$ and $g_{2}$, respectively, occur in the bracket $B$. The degree of $B$ is equal to the value of $\sum_{i=0}^{2} \delta^{i}(B)$. The Sussmann condition for small time local controllability is essentially that the so-called bad brackets, the brackets with $\delta^{0}(B)$ odd and $\delta^{1}(B), \delta^{2}(B)$ even, must be a linear combination of good (i.e. not of the bad type) brackets of lower degree at the equilibrium. The degree of a bad bracket must necessarily be odd. The only bad bracket of degree one is $f$ which vanishes at any equilibrium. The bad brackets of degree three are brackets with $\delta^{0}=1$ and $\delta^{i}=2, i=1$ or 2 , and all are identically zero vector fields. It follows that the Sussman condition is satisfied at $x^{e}$. Hence the system is small time locally controllable at $x^{e}$.

Since the system is real analytic, the above controllability results imply the existence of piecewise analytic feedback laws [18] which asymptotically stabilize the closed loop system to a given $x^{e}$. In the next section, guided by these results, we will focus on designing asymptotically stabilizing discontinuous feedback laws for the system.

\section{Discontinuous Feedback Laws}

In this section, we will consider the problem of designing feedback control laws of the form $u=u(x)$ for the system (8)-(13). As discussed in the previous section, the system cannot be asymptotically stabilized to an equilibrium using any time-invariant continuous feedback. Therefore, we restrict our consideration to designing time-invariant discontinuous feedback laws.

Note that the problem of stabilizing the system to a given equilibrium $x^{e} \in \mathbf{M}^{e}$ can be reduced to the problem of stabilizing the system to the origin via an appropriate state transformation. Hence, without loss of generality, we focus only on the problem of feedback stabilization to the origin, i.e. $x^{e}=0$.

We will first study the problem of stabilizing the following reduced order system, which is obtained by considering the subsystem (8)-(11) and letting $\left(x_{5}, x_{6}\right)$ be the control variables $\left(v_{1}, v_{2}\right)$ :

$\dot{x}_{1}=v_{1}$

$\dot{x}_{2}=v_{2}+x_{3} v_{1}$

$\dot{x}_{3}=x_{4}-x_{2} v_{1}$

$\dot{x}_{4}=-\alpha x_{4}-\beta v_{1} v_{2}$.

The idea that will be employed is based on first transforming the reduced system (17)-(20) into a discontinuous one by applying a discontinuous coordinate transformation, e.g. by applying a $\sigma$-process (see e.g. [1]).
From the analytical point of view, the $\sigma$-process, also termed as the process of resolution of singularities, consists of a rational coordinate transformation.

Consider the reduced system (17)-(20). Restricting consideration to $x_{1} \neq 0$, apply the $\sigma$-process

$y=x_{1}, z_{1}=x_{2}, z_{2}=\frac{x_{3}}{x_{1}}, z_{3}=\frac{x_{4}}{x_{1}}$

to obtain

$\dot{y}=v_{1}$,

$\dot{z}_{1}=v_{2}+y z_{2} v_{1}$,

$\dot{z}_{2}=z_{3}-\frac{z_{1}+z_{2}}{y} v_{1}$

$\dot{z}_{3}=-\alpha z_{3}-\frac{z_{3}+\beta v_{2}}{y} v_{1}$

Clearly, the feedback control law

$v_{1}=-k y$,

$v_{2}=-l z$,

where $k>0$ and $l=\left(l_{1} l_{2} l_{3}\right)$ are the gains, yields the reduced closed-loop system

$\dot{y}=-k y$,

$\dot{z}_{1}=-l_{1} z_{1}-l_{2} z_{2}-l_{3} z_{3}-k y^{2} z_{2}$,

$\dot{z}_{2}=k z_{1}+k z_{2}+z_{3}$

$\dot{z}_{3}=-k \beta l_{1} z_{1}-k \beta l_{2} z_{2}+\left(k-\alpha-k \beta l_{3}\right) z_{3}$.

The $z$-dynamics can be rewritten as

$\dot{z}=\left(A_{1}+A_{2}(t)\right) z$

where

$A_{1}=\left(\begin{array}{ccc}-l_{1} & -l_{2} & -l_{3} \\ k & k & 1 \\ -k \beta l_{1} & -k \beta l_{2} & k-\alpha-k \beta l_{3}\end{array}\right)$,
$A_{2}(t)=\left(\begin{array}{ccc}0 & -k y_{0}^{2} e^{-2 k t} & 0 \\ 0 & 0 & 0 \\ 0 & 0 & 0\end{array}\right)$.

It can be easily seen that if $k \neq \alpha$, the spectrum of the matrix $A_{1}$ can be assigned arbitrarily through the gain matrix $l$. Clearly, the $y$-dynamics is globally exponentially stable at $y=0$. Moreover, since the matrix $A_{2}(t)$ given by (33) goes to zero as $t \rightarrow \infty$ and

$\int_{0}^{\infty}\left\|A_{2}(t)\right\| d t<\infty$,

the $z$-dynamics can also be rendered globally exponentially stable at the origin $z=0$ by selecting $l=\left(\begin{array}{lll}l_{1} & l_{2} & l_{3}\end{array}\right)$ 
such that the matrix $A_{1}$ given by (32) is a Hurwitz matrix (see [15], Section 4.2.2).

Note that in the original coordinates the controls (25)-(26) take the form

$v_{1}\left(x_{1}, x_{2}, x_{3}, x_{4}\right)=-k x_{1}$,

$v_{2}\left(x_{1}, x_{2}, x_{3}, x_{4}\right)=-l_{1} x_{2}-l_{2} \frac{x_{3}}{x_{1}}-l_{3} \frac{x_{4}}{x_{1}}$,

and the reduced closed-loop system is given by

$\dot{x}_{1}=-k x_{1}$

$\dot{x}_{2}=-l_{1} x_{2}-l_{2} \frac{x_{3}}{x_{1}}-l_{3} \frac{x_{4}}{x_{1}}-k x_{1} x_{3}$,

$\dot{x}_{3}=x_{4}+k x_{1} x_{2}$

$\dot{x}_{4}=-k \beta l_{2} x_{3}-\left(k \beta l_{3}+\alpha\right) x_{4}-k \beta l_{1} x_{1} x_{2}$.

We now present the following result.

Proposition 3: Consider the reduced closed-loop system (36)-(39) with $k>0, k \neq \alpha$, and $l=\left(l_{1} l_{2} l_{3}\right)$ selected such that the matrix $A_{1}$ given by equation (32) is a Hurwitz matrix. Let $\left(x_{10}, x_{20}, x_{30}, x_{40}\right)$ denote an initial condition with $x_{10} \neq 0$. Then the following hold.

(i) The trajectory $\left(x_{1}(t), x_{2}(t), x_{3}(t), x_{4}(t)\right)$ is bounded for all $t \geq 0$ and converges exponentially to zero.

(ii) The control $\left(v_{1}(t), v_{2}(t)\right)$ is bounded for all $t \geq 0$ and converges exponentially to zero.

Proof: Consider the reduced closed-loop system (36)(39) and denote by $\left(x_{10}, x_{20}, x_{30}, x_{40}\right)$ an initial condition with $x_{10} \neq 0$.

(i) Clearly, we have $x_{1}(t)=x_{10} e^{-k t}$. Since $x_{10} \neq 0$, $x_{1}(t) \neq 0, \forall t \in[0, \infty)$. Under the stated assumptions, the reduced closed-loop system in the transformed coordinates is globally exponentially stable. Thus, the variables $x_{1}(t), x_{2}(t), x_{3}(t) / x_{1}(t), x_{4}(t) / x_{1}(t)$ converge exponentially to zero. It follows that the trajectory $\left(x_{1}(t), x_{2}(t), x_{3}(t), x_{4}(t)\right)$ is bounded for all $t \geq 0$ and converges exponentially to zero.

(ii) From the above discussion, each term in the control $v$ given by (34)-(35) consists of bounded terms which converge exponentially to zero. Hence, the result follows.

Remark 1: The above result demonstrates that for initial conditions satisfying $x_{10} \neq 0$, the feedback control law (34)-(35) is well-defined for all $t \geq 0$. Moreover, it drives the system (36)-(39) to the origin, while avoiding the set

$N=\left\{\left(x_{1}, x_{2}, x_{3}, x_{4}\right) \mid x_{1}=0,\left(x_{1}, x_{2}, x_{3}, x_{4}\right) \neq 0\right\}$.

Clearly, one can use a finite time feedback law [9] to move the system away from $N$.

We now return to the problem of asymptotic stabilization of the system (8)-(13) with $u_{1}$ and $u_{2}$, instead of $x_{5}$ and $x_{6}$, as control inputs. Note that since $u_{1}=\dot{x}_{5}$ and $u_{2}=\dot{x}_{6}$, the problem corresponds to the classical situation where integrators are added at the input level.

Again restrict consideration to $x_{1} \neq 0$ and consider the following controller

$$
\begin{aligned}
& u_{1}(x)=-K\left(x_{5}-v_{1}\left(x_{1}, x_{2}, x_{3}, x_{4}\right)\right)+s_{1}(x), \\
& u_{2}(x)=-L\left(x_{6}-v_{2}\left(x_{1}, x_{2}, x_{3}, x_{4}\right)\right)+s_{2}(x),
\end{aligned}
$$

where $v_{1}\left(x_{1}, x_{2}, x_{3}, x_{4}\right)$ and $v_{2}\left(x_{1}, x_{2}, x_{3}, x_{4}\right)$ denote the feedback controls (34)-(35) for the reduced system; and $s_{1}(x)$ and $s_{2}(x)$ correspond to their time derivatives along the trajectories of the system (8)-(13):

$$
\begin{aligned}
s_{1}(x)= & -k x_{5} \\
s_{2}(x)= & -l_{1}\left(x_{6}+x_{3} x_{5}\right)-l_{2} \frac{x_{4}-x_{2} x_{5}}{x_{1}}+l_{3} \frac{\alpha x_{4}+\beta x_{5} x_{6}}{x_{1}} \\
& +l_{2} \frac{x_{3} x_{5}}{x_{1}^{2}}+l_{3} \frac{x_{4} x_{5}}{x_{1}^{2}} .
\end{aligned}
$$

Now assume that the control parameters are selected such that $k, l_{1}, l_{2}$ and $l_{3}$ satisfy the conditions of Proposition 3 and $K>k, L>0$. Consider the coordinate transformation

$y=x_{1}, z_{1}=x_{2}, z_{2}=\frac{x_{3}}{x_{1}}, z_{3}=\frac{x_{4}}{x_{1}}$

$w_{1}=x_{5}+k x_{1}, w_{2}=x_{6}+l_{1} x_{2}+l_{2} \frac{x_{3}}{x_{1}}+l_{3} \frac{x_{4}}{x_{1}}$.

It can be shown that in the above coordinates the closedloop system can be written as

$$
\begin{aligned}
& \dot{y}=-k y+w_{1}, \\
& \dot{z}=\left(A_{1}+\tilde{A}_{2}(t)\right) z+h(t), \\
& \dot{w}_{1}=-K w_{1}, \\
& \dot{w}_{2}=-L w_{2},
\end{aligned}
$$

where $A_{1}$ is the matrix given by (32) and

$$
\begin{aligned}
& \tilde{A}_{2}(t)=\left(\begin{array}{ccc}
0 & r_{1}(t) & 0 \\
-r_{2}(t) & r_{2}(t) & 0 \\
\beta l_{1} r_{2}(t) & \beta l_{2} r_{2}(t) & \left(\beta l_{3}-1\right) r_{2}(t)
\end{array}\right), \\
& r_{1}(t)=\left(e^{-k t} y_{0}+\frac{e^{-K t}-e^{-k t}}{k-K} w_{10}\right) \\
& \left(-k e^{-k t} y_{0}+\left(e^{-K t}-e^{-k t}\right) w_{10}\right) \text {, } \\
& r_{2}(t)=\left(e^{-k t} y_{0}+\frac{e^{-K t}-e^{-k t}}{k-K} w_{10}\right)^{-1} e^{-K t} w_{10} \\
& \left.h(t)=\left(\begin{array}{ccc}
1 & 0 & \beta\left(k-r_{2}(t)\right.
\end{array}\right)\right)^{\prime} e^{-L t} w_{20} .
\end{aligned}
$$

Clearly, $\left(y, w_{1}, w_{2}\right)$-dynamics is globally exponentially stable at $\left(y, w_{1}, w_{2}\right)=(0,0,0)$. Moreover, it can be shown that if $w_{10}=k y_{0}$ (or, equivalently, $x_{50}=0$ ), then $\tilde{A}_{2}(t)$ and $h(t)$ go to zero as $t \rightarrow \infty$; and

$$
\int_{0}^{\infty}\left\|A_{2}(t)\right\| d t<\infty, \quad \int_{0}^{\infty}\|h(t)\| d t<\infty .
$$


It follows that, for any initial condition $\left(y_{0}, z_{0}, w_{10}, w_{20}\right)$ satisfying $y_{0} \neq 0$ and $w_{10}=k y_{0}$, both the trajectory $\left(y(t), z(t), w_{1}(t), w_{2}(t)\right)$ and the control $\left(u_{1}(t), u_{2}(t)\right)$ are bounded for all $t \geq 0$ and converge exponentially to zero.

We now present the following result.

Proposition 4: Consider the system (8)-(13) with the feedback controls (40)-(41), where the control parameters are selected such that $k, l_{1}, l_{2}$ and $l_{3}$ satisfy the conditions of Proposition 3 and $K>k, L>0$. Let $x_{0}=\left(x_{10}, x_{20}, x_{30}, x_{40}, x_{50}, x_{60}\right)$ denote an initial condition satisfying $x_{10} \neq 0$ and $x_{50}=0$. Then the following hold.

(i) The trajectory $\left(x_{1}(t), x_{2}(t), x_{3}(t), x_{4}(t), x_{5}(t), x_{6}(t)\right)$ is bounded for all $t \geq 0$ and converges exponentially to zero.

(ii) The control $\left(u_{1}(t), u_{2}(t)\right)$ is bounded for all $t \geq 0$ and converges exponentially to zero.

Remark 2: Note that the above choice of the feedback control guarantees that the $x_{1}$-dynamics satisfies the second-order linear dynamics

$\ddot{x}_{1}+(K+k) \dot{x}_{1}+K k x_{1}=0$.

It can be easily seen that if $x_{10} \neq 0$ and $x_{50}=0$, then $x_{1}(t) \neq 0, \forall t \in[0, \infty)$. Thus, for all initial conditions satisfying $x_{10} \neq 0$ and $x_{50}=0$, the feedback control law (40)-(41) is well-defined for all $t \geq 0$. Moreover, it drives the system (8)-(13) to the origin, while avoiding the set

$N^{\prime}=\left\{x \in \mathbf{R}^{6} \mid x_{1}=0, x \neq 0\right\}$.

Clearly, one can use a finite time feedback control law to move the system to a state satisfying the conditions of Proposition 4. For example,

$u_{1}=-\left|x_{1}-\epsilon\right|^{a} \operatorname{sign}\left(x_{1}-\epsilon\right)-\left|x_{5}\right|^{b} \operatorname{sign}\left(x_{5}\right)$,

$u_{2}=0$,

where $b \in(0,1), a>b /(2-b)$ and $\epsilon \neq 0$ are constants, can be used to transfer the system to a state statisfying the conditions of Proposition 4 in finite time [9].

\section{Example}

We illustrate the results of the paper with a simulation example of a surface vessel with two independent propellers as shown in Figure 1. The system parameters are given by

$m_{11}=200 \mathrm{~kg}, m_{22}=250 \mathrm{~kg}, m_{33}=80 \mathrm{~kg} \cdot \mathrm{m}^{2}$,

$d_{11}=70 \mathrm{~kg} \cdot \mathrm{s}^{-1}, d_{22}=100 \mathrm{~kg} \cdot \mathrm{s}^{-1}, d_{33}=50 \mathrm{~kg} \cdot \mathrm{m}^{2} \cdot \mathrm{s}^{-1}$.

A computer implementation of the discontinuous feedback control law specified in Proposition 4 was used to asymptotically stabilize the origin. The gains were chosen as

$k=1, l=(3.0208,2.8125,0.0990), K=2, L=2$.

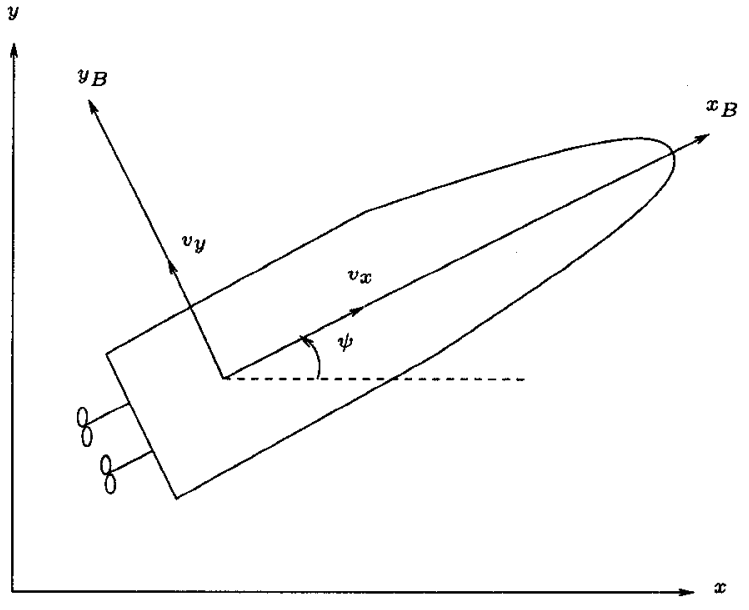

Figure 1: Model of a Surface Vessel.

Note that the above choice of the gain matrix $l$ locates the eigenvalues of the matrix $A_{1}$ given by equation (32) at $(-0.5,-0.5,-0.5)$. The results of the simulation for a sample initial condition given by $\left(x_{1}, x_{2}, x_{3}, x_{4}, x_{5}, x_{6}\right)$ $=(0.5 \pi, 1,-1,0,0,0)$ (or equivalently $\left(x, y, \psi, v_{x}, v_{y}, \omega_{z}\right)$ $=(1,1,0.5 \pi, 0,0,0))$ are shown in Figures 2-4. Figure 2 shows the time responses of the configuration variables $x, y$ and $\psi$. The time responses for the velocities $v_{x}, v_{y}$ and $\omega_{z}$ and the controls $F_{x}$ and $T_{z}$ are shown in Figures 3 and 4, respectively. Exponential convergence of the closed-loop state and control trajectories can be observed from the results of the simulation.

\section{Conclusions}

Following a controllability and stabilizability analysis, time-invariant discontinuous feedback control laws have been derived for the asymptotic stabilization of a surface vessel with two independent thrusters. Boundedness and convergence of the closed-loop state and control trajectories have been demonstrated. The effectiveness of the proposed feedback control laws has been illustrated through a simulation example.

\section{Acknowledgement}

The author wishes to acknowledge the support provided by the Dutch Institute of Systems and Control that enabled his visit to the University of Twente during the academic year 1995-1996.

\section{References}

[1] V.I. Arnold, Geometrical Methods in the Theory of Ordinary Differential Equations, Springer-Verlag, New York Inc., 1983.

[2] A. Astolfi, "Exponential Stabilization of Nonholonomic Systems via Discontinuous Control," Nonlinear Control System Design Symposium (NOLCOS), IFAC, Tahoe City, 1995, pp.741-746. 
[3] N.S. Bedrossian, "Nonlinear Control of an Underactuated Two-Link Manipulator," Proceedings of IEEE Conference on Decision and Control, 1993, San Antonio, Texas, pp.2234-2238.

[4] A.M. Bloch, M. Reyhanoglu and N.H. McClamroch, "Control and Stabilization of Nonholonomic Dynamic Systems," IEEE Transactions on Automatic Control, 37(11), 1992, pp.1746-1757.

[5] R.W. Brockett, "Asymptotic Stability and Feedback Stabilization," in Differential Geometric Control Theory, R.W. Brockett, R.S. Millman and H.J. Sussmann, eds., Birkhauser, Boston, 1983, pp.181191.

[6] O. Egeland, M. Dalsmo and O.J. Sordalen, "Feedback Control of a Nonholonomic Underwater Vehicle with Constant Desired Configuration," to appear in International Journal of Robotics Research, 1996.

[7] T.I. Fossen, Guidance and Control of Ocean Vehicles, John Wiley and Sons Ltd., 1994.

[8] Y.-L. Gu, "A Direct Adaptive Control Scheme for Underactuated Dynamic Systems," Proceedings of IEEE Conference on Decision and Control, 1993, San Antonio, Texas, pp.1625-1627.

[9] V.T. Haimo, "Finite Time Controllers," SIAM J. Control and Optimization, 24(4), 1986, pp.760-770.

[10] H. Nijmeijer and A.J. van der Schaft, Nonlinear Dynamical Control Systems, New York: SpringerVerlag, 1990.

[11] G. Oriolo and Y. Nakamura, "Control of Mechanical Systems with Second-Order Nonholonomic Constraints: Underactuated Manipulators," Proceedings of IEEE Conference on Decision and Control, 1991, Brighton, UK, pp.2398-2403.

[12] M. Reyhanoglu, "On the Stabilization of a Class of Nonholonomic Systems Using Invariant Manifold Technique," Proceedings of IEEE Conference on Decision and Control, 1995, New Orleans, LA, pp.2125-2126.

[13] M. Reyhanoglu, A.J. van der Schaft, N.H. McClamroch and I. Kolmanovsky, "Nonlinear Control of a Class of Underactuated Systems," Proceedings of IEEE Conference on Decision and Control, 1996.

[14] D. Seto and J. Baillieul, "Control Problems in Super-Articulated Mechanical Systems," IEEE Transactions on Automatic Control, 39(12), 1994, pp.2442-2453.

[15] J-J.E. Slotine and W. Li, Applied Nonlinear Control, Prentice-Hall Inc., Englewood Cliffs, New Jersey, 1991.

[16] M.W. Spong, "Energy Based Control of a Class of Underactuated Mechanical Systems," Proceedings of IFAC World Congress, San Francisco, California, 1996, pp.431-435.
[17] H.J. Sussmann, "A General Theorem on Local Controllability," SIAM Journal on Control and Optimization, 25(1), 1987, pp.158-194.

[18] H.J. Sussmann, "Subanalytic Sets and Feedback Control," J. Differential Equations, 31, 1979, pp.3152.

[19] K.Y. Wichlund, O.J. Sordalen and O. Egeland, "Control of Vehicles with Second-Order Nonholonomic Constraints: Underactuated Vehicles," Proceedings of European Control Conference, 1995, Rome, Italy, pp.3086-3091.

[20] J. Zabczyk, "Some Comments on Stabilizability," Applied Math. Optimization, 19, 1989, pp.1-9.

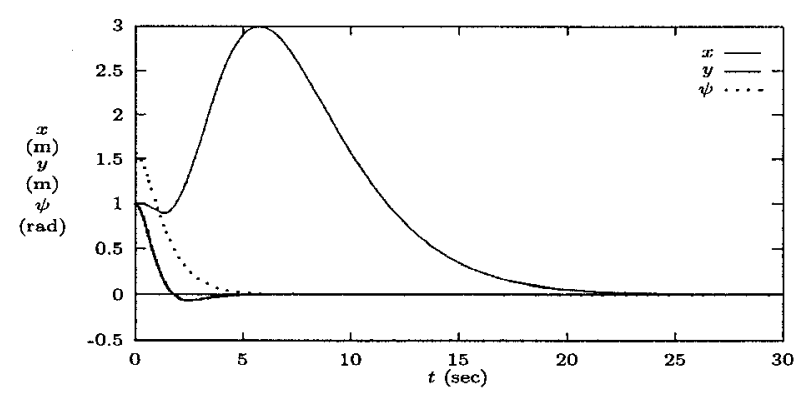

Figure 2: Configuration Variables $x, y$ and $\psi$.

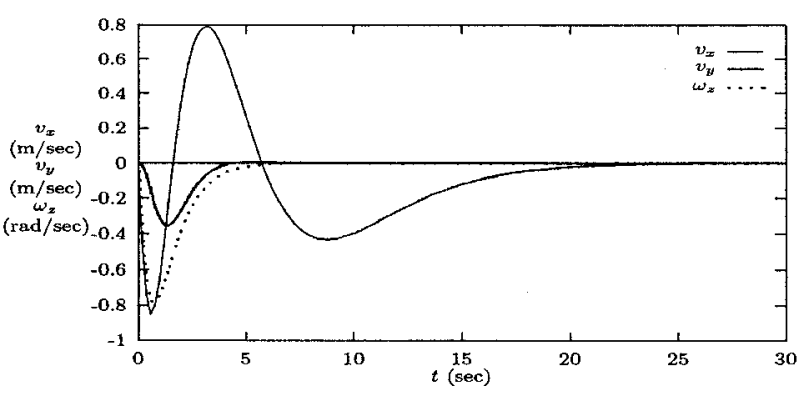

Figure 3: Velocity Variables $v_{x}, v_{y}$ and $\omega_{z}$.

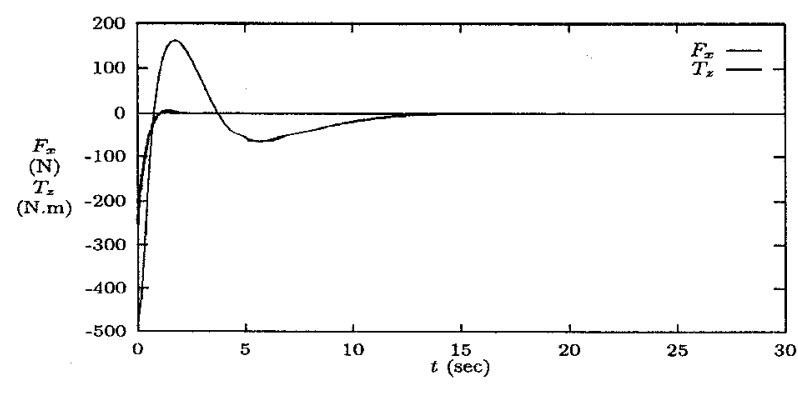

Figure 4: Controls $F_{x}$ and $T_{z}$. 